\title{
Plasma cell free DNA methylation markers for hepatocellular carcinoma surveillance in patients with cirrhosis: a case control study
}

Jörn Lewin ${ }^{1 \dagger}$, Denise Kottwitz ${ }^{1 \dagger}$, Johanna Aoyama' ${ }^{1}$, Theo deVos ${ }^{2 *} \mathbb{C}$, Jorge Garces ${ }^{1}$, Oliver Hasinger ${ }^{1}$, Stefanie Kasielke ${ }^{1}$, Florian Knaust ${ }^{1}$, Preeti Rathi' ${ }^{1}$, Sebastian Rausch' ${ }^{1}$, Gunter Weiss ${ }^{1}$, Alexander Zipprich ${ }^{3}$, Edward Mena ${ }^{4}$ and Tse-Ling Fong ${ }^{5}$

\begin{abstract}
Background: Hepatocellular carcinoma (HCC) is the leading cause of death in patients with cirrhosis, primarily due to failed early detection. HCC screening is recommended among individuals with cirrhosis using biannual abdominal ultrasound, for earlier tumor detection, administration of curative treatment, and improved survival. Surveillance by imaging with or without biomarkers such as alpha-fetoprotein (AFP) remains suboptimal for early stage HCC detection. Here we report on the development and assessment of methylation biomarkers from liquid biopsies for HCC surveillance in cirrhotic patients.
\end{abstract}

Methods: DNA methylation markers including the HCCBloodTest (Epigenomics AG) and a DNA-methylation panel established by next generation sequencing (NGS) were assessed using a training/testing design. The NGS panel algorithm was established in a training study (41 HCC patients; 46 cirrhotic non-HCC controls). For testing, plasma samples were obtained from cirrhotic patients (Child class A or B) with (60) or without (103) early stage HCC (BCLC stage 0, A, B). The assays were then tested using blinded sample sets and analyzed by preset algorithms.

Results: The HCCBloodTest and the NGS panel exhibited $76.7 \%$ and $57 \%$ sensitivities at $64.1 \%$ and $97 \%$ specificity, respectively. In a post-hoc analysis, a combination of the NGS panel with AFP $(20 \mathrm{ng} / \mathrm{mL})$ achieved $68 \%$ sensitivity at 97\% specificity $(A \cup C=0.9)$.

Conclusions: Methylation biomarkers in cell free plasma DNA provide a new alternative for HCC surveillance. Multiomic panels comprising DNA methylation markers with other biological markers, such as AFP, provide an option to further increase the overall clinical performance of surveillance via minimally invasive blood samples.

Trial Registration: Test set study_ClinicalTrials.gov (NCT03804593) January 11, 2019, retrospectively registered.

Keywords: Carcinoma, Hepatocellular, Liver cirrhosis, Early detection of cancer, Biomarker, Cell-free nucleic acids, DNA methylation

*Correspondence: theo.devos@epigenomics.com

${ }^{\dagger} J$ J̈rn Lewin and Denise Kottwitz contributed equally to this work and shared first authorship

${ }^{2}$ Epigenomics Inc., 11055 Flintkote Ave, Suite A, San Diego, CA 92121, USA

Full list of author information is available at the end of the article

\section{Background}

Hepatocellular carcinoma (HCC) is the 5th most common cancer in men, and 9th in women worldwide with an incidence of more than 840,000 cases and 780,000 deaths annually (2018) [1]. In the US an estimated 42,000 new cases and 30,000 deaths due to HCC will occur in 2020 [2]. Most HCC cases occur in patients with cirrhosis, a 
condition affecting an estimated 4.5 million Americans [3]. The diagnosis of HCC at an early stage offers the chance of curative treatment whereas regional and metastatic disease is associated with a 5-year survival rates of $11 \%$ and $3 \%$ respectively [2]. To identify $\mathrm{HCC}$ at an early treatable stage, the American Association for the Study of Liver Diseases (AASLD) recommends surveillance of adults with cirrhosis by ultrasound with or without alpha-fetoprotein (AFP) every 6 months [4].

The AASLD guideline recommendation regarding AFP was based in part on an improvement in sensitivity of HCC detection when AFP was used in conjunction with ultrasound (US). Though the data is limited, performance of combined US + AFP is still not optimal for early detection, with a reported sensitivity of $63 \%$ at a pooled specificity of $84 \%[5,6]$. Given the size of the at risk cirrhotic population, the effort required for guideline recommended surveillance, the current suboptimal performance of recommended surveillance methods, and the low surveillance rates, there is a significant clinical need for novel minimally invasive testing to aid in the detection of $\mathrm{HCC}$ at an early stage. Efforts to address this need include discovery and development of novel biomarkers [7], and diagnostic algorithms combining biomarker data and patient features to improve performance. As an example, patient sex and age combined with measurement of Lens culinaris agglutinin-reactive AFP (AFP-L3), AFP, and des- $\gamma$-carboxyprothrombin (DCP) was used to produce the diagnostic GALAD score. External validation for one model resulted in improved sensitivity of detection to the $>90 \%$ though specificity dropped to $62 \%$ [8]. When used in combination with ultrasound (GALADUS) detection is further enhanced, though this observation requires further external validation [9].

A recent innovation in the cancer diagnostics field has been the analysis of genetic or epigenetic cancer markers in patient plasma or serum. This approach, termed liquid biopsy, is becoming a standard clinical tool for the identification [10] and classification of cancer as well as an aid in treatment selection $[11,12]$. This has been achieved by measuring circulating tumor DNA (ctDNA) [13, 14] in patient blood either by detection of genetic changes such as single nucleotide polymorphism (SNP) patterns or somatic gene mutations as in the recent example of the TERT C228T promoter mutation [15], or by identifying tumor associated DNA methylation patterns in cell free DNA (cfDNA) [16]. For example, previous studies showed that the presence of methylated SEPTIN9 (mSEPT9) DNA in plasma cfDNA was correlated with the occurrence of HCC in patients with cirrhosis [17, 18]. The SEPTIN9 gene encodes Septin-9, a member of the conserved septin family of GTP-binding proteins that function in key processes including vesicle trafficking, apoptosis, cytoskeletal remodeling and cell division [19]. The Septin-9 protein also acts as a tumor suppressor, regulating orderly and controlled cell growth.

The primary objective of this study was to determine the performance of mSEPT9 (commercially known as HCCBloodTest) and a novel methylated DNA biomarker panel for HCC surveillance. These assays were tested using plasma cfDNA from well characterized cirrhotic patients with and without early stage HCC Barcelona Clinic Liver Cancer (BCLC, stages 0, A, B) as the objective of HCC surveillance is the detection of patients with early stage disease. In addition, we report the results of a post-hoc analyses combining the NGS panel of gene methylation markers with AFP measurement for HCC surveillance in this patient population.

\section{Methods \\ Design}

The study was designed to determine the performance of mSEPT9 and a novel next generation sequencing (NGS) DNA methylation panel (without mSEPT9) as surveillance biomarkers for HCC detection in patients with cirrhosis. The biomarker panel was first established and the interpretive algorithm trained using plasma DNA samples from cirrhotic patients with and without cirrhosis. mSEPT9 was used in the HCCBloodTest format (Epigenomics AG Berlin Germany) following the instructions for use for the kit. The performance of the methylation panel and HCCBloodTest were then tested using plasma samples from a cross-sectional case control study of well characterized cirrhotic patients with no HCC or early stage HCC. Post hoc analyses were performed to assess the performance of the panel in combination with AFP measurement in this population.

\section{Patients}

NGS Marker Discovery and Training: Sample collection protocols were approved by local Institutional Review Boards and all patients provided informed consent to participate in the studies. Plasma samples from 41 cirrhotic subjects with HCC and 46 subjects with cirrhosis who were negative for $\mathrm{HCC}$ were used for the training set (University Halle-Wittenberg, ethical approval No. 2012-5).

For testing, subjects were enrolled under a cross sectional case control study design registered on ClinicalTrials.gov (NCT03804593). Patients were enrolled at the University of Southern California (USC) Keck Medical Center and the California Liver Research Institute. The study collection protocol was approved by the Institutional Review Boards at both sites and patients provided written informed consent prior to study participation. Patients included men and women 18 years or older with 
Child class A or B scores. Exclusion criteria are provided in the Additional file 1. In addition to plasma sample collection, AFP measurements were also obtained for all subjects.

Controls (Group 1) included patients with a diagnosis of cirrhosis and no $\mathrm{HCC}$ as confirmed by 4 phase abdominal contrast-enhanced MRI or CT imaging performed $\leq 90$ days prior to the date of consent or an abdominal contrast-enhanced MRI performed $\leq 45$ days after enrollment. Patients had either no lesions or lesions with a liver imaging reporting and data system (LI-RADS) score of LR-1 or LR-2. All abdominal imaging was interpreted by one central radiologist. A total of 103 patients with Child class A or B met enrollment criteria for Group 1 and provided a sufficient plasma sample for the study. 102 of these were sufficient for additional research.

Cases (Group 2) included patients with a diagnosis of HCC that was confirmed by use of a 4-phase abdominal MRI or CT imaging performed $\leq 90$ days prior to the date of consent or by use of a 4-phase abdominal MRI performed $\leq 45$ days after enrollment with a LI-RADS category of LR-5 and/or biopsy with histopathology. All abdominal imaging was interpreted by one central radiologist. A total of 60 patients fulfilled the enrollment criteria and Barcelona Clinic Liver Cancer (BCLC) stage criteria (0/A/B) for Group 2 and provided a sufficient plasma sample.

\section{Sample preparation}

Blood samples were drawn in EDTA plasma tubes, and plasma was prepared by centrifugation and further cleared by a second centrifugation. Plasma aliquots were stored at $-70{ }^{\circ} \mathrm{C}$ until processing.

Bisulfite treated DNA (bisDNA) was prepared from plasma with reagents from the commercially available Epi BiSKit (Epigenomics AG, Berlin) [20] using an automated protocol on the Tecan Evo 200 liquid handling platform (Männedorf, $\mathrm{CH}$ ) [21]. In order to ensure that all samples from the same patient were comparable, multiple plasma samples from an individual patient were pooled and equally distributed into $3.5 \mathrm{ml}$ aliquots, processed in parallel and stored at $-20^{\circ} \mathrm{C}$.

\section{Multiplex marker panel}

The bisulfite targeted multiplex NGS panel as used for training, consisted of two methylation unspecific control DNA targets and 17 targeted methylation specific marker candidates (including SEPTIN9), ranging in amplicon size from 63 to 105 bases. The marker candidates used in the training panel originated from three different sources: 1 . Six previously described cancer markers (including mSept9 and mRASSF2). 2. Five marker candidates originated from Epigenomics' internal discovery by differential methylation hybridization (DMH) [22]. 3. Six candidates identified by in-silico discovery aiming for specific markers methylated in HCC but unmethylated in blood and preferably also unmethylated in other (solid) cancers, using public data from different sources. The final panel used in testing comprised the same markers, excluding SEPTIN9.

\section{Multiplex PCR for NGS}

The PCR was set up as one single reaction per sample using bisulfite DNA template from an equivalent of about $1 \mathrm{ml}$ plasma in a ready to use multiplex PCR reaction (QIAGEN $^{\circledR}$ Multiplex PCR) according to the manufacture's recommended protocol. PCR primers were modified with a 5 'phosphate for NGS library preparation. The multiplex PCR profile used a protocol as follows: denaturation at $94{ }^{\circ} \mathrm{C}$ for $30 \mathrm{~s}$, annealing at $56{ }^{\circ} \mathrm{C}$ for $90 \mathrm{~s}$, extension for $30 \mathrm{~s}$ at $72{ }^{\circ} \mathrm{C} ; 45$ cycles.

\section{Library preparation and 2nd generation Sequencing}

NGS library preparation was done according to the Illumina TruSeqNano DNA library preparation protocol [TruSeq ${ }^{\circledR}$ NanoDNALibraryPrep ReferenceGuide, Illumina] with the following modifications: The DNA fragmentation and end repair steps were skipped and DNA purification steps were adapted to isolate shorter DNA fragments. $15 \mu \mathrm{l}$ of each multiplex PCR was purified using a magnetic beads/DNA ratio of 0.8 in the presence of $20 \%$ isopropanol. Purification steps after adenylation, adapter ligation and enrichment PCR were done with a magnetic beads/DNA ratio of 0.6 also in the presence of $20 \%$ isopropanol. The prepared libraries were quantified with the Qubit dsDNA HS Assay Kit (Thermo Fisher Scientific). Sequencing was done with MiSeq Reagent Kit v2 using a read length of $300 \mathrm{bp}$ and targeting $200 \mathrm{k}$ reads per sample.

\section{NGS raw data interpretation}

Paired Fastq files were trimmed to insertions between sequencing adaptors, and paired sequences were merged using flash [23]. All further data analysis was done in $R$ [24] based on proprietary code using Rcpp [25] to increase processing speed and comprised the following steps: sequences were filtered for those flanked by primers on both sides reflecting molecules amplified by PCR, called Inserts. Inserts containing more cytosine than guanine outside of the $\mathrm{CpG}$ context were turned to their reverse complement to enable easy assessment of methylation by taking cytosine positions of CpGs into account exclusively. Such inserts were aligned to reference sequences of the assays to assess DNA-methylation: For each assay/sample combination any methylation 
pattern at CpG sites was assessed by counting occurrence of cytosines and thymidines at $\mathrm{CpG}$ positions. Comethylation within a single insert read was defined by cytosine in all or all except one CpG position (allowing an exception of one CpG to be different due to any error or SNP at a single CpG site) [26]. Quantitative co-methylation measured as the normalized number of co-methylated fragments of a marker in a sample was calculated as the number of comethylated insert sequences divided by the total number of all inserts found for a sample, normalized by the length of the sequences (in the following simply referred to as co-methylation).

\section{Training the NGS panel}

Training was performed by a simple, robust method applicable to small sample sets: marker candidate performance was characterized by receiver operating characteristic (ROC) differentiating groups of cirrhotic patients without $\mathrm{HCC}$ and those with $\mathrm{HCC}$ using quantitative co-methylation. $\mathrm{N}$ marker candidates with an area under the curve (AUC) of 0.7 were defined as usable markers. For each of the $\mathrm{N}$ markers a co-methylation cutoff was determined at a specificity of 0.9 that was used to determine whether a single marker was classified positive or negative. Marker panel measurements for a sample were defined as number of $n$ positive markers. The marker panel characteristic was described by using $n / N$ e.g. for ROC characteristics. The set of markers and their comethylation cutoffs from the training set were defined as the training result and stored in an $\mathrm{R}$ object.

\section{Assay performance}

\section{mSEPT9/HCCBloodTest}

For the HCCBloodTest, bisDNA from each patient sample was analyzed as PCR triplicates on an ABI 7500 FAST Dx. The final test results of the HCCBloodTest were derived from the triplicate results by means of the algorithm defined in the respective Instructions for Use [27].

\section{NGS panel}

Bisulfite DNA from plasma samples were processed, measured and assessed blinded. For each sample each marker was binarized to be either positive or negative using the trained comethylation cutoffs leading to scores of $n$ [0:7] positive markers for each sample. Patient group identity was then un-blinded and performance of the panel described as AUC and sensitivity/specificity at a cutoff of $n+/ 7$.

\section{Post-hoc analyses}

Additional analyses were performed assessing a combination of mSEPT9 and AFP $(20 \mathrm{ng} / \mathrm{mL})$ as well as for the multi-marker NGS panel, including an alternative simple additive algorithm and a combination of the NGS results with AFP $(20 \mathrm{ng} / \mathrm{mL})$ to form a multiomic panel.

\section{Statistical analysis}

Analysis was primarily descriptive and performed using standard libraries of the R environment [24]. If not stated otherwise, 95\% confidence intervals are reported, and statistical tests were conducted at significance level 0.05 .

\section{Results}

Training samples included 46 cases and 41 controls. For testing, 61 patients with cirrhosis and HCC (cases) and 104 patients with cirrhosis and no detectable HCC (controls) were enrolled (Table 1). Excluded patients included one control patient with Child $\mathrm{C}$ cirrhosis; one patient with HCC who had a stage D (advanced) cancer by BCLC criteria; and for one control patient there was insufficient plasma for analysis using the additional NGS panel. Patient information including age, sex, Child class, etiology of cirrhosis and for HCC cases information on nodules and BCLC stage of cancer is summarized in Table 1. Additional patient information is provided in Additional file 1: Figure S1. There was a predominance of males among patients with HCC and they were older. The underlying etiologies of cirrhosis were non-alcoholic steatohepatitis (NASH), chronic viral infection (HBV, HCV) and alcoholic liver disease (ALD).

\section{Panel training results}

From the 16 marker candidates assessed in the targeted multiplex PCR panel, seven markers could be identified based on $A U C \geq 0.7$ : mASCL2, mLDHB, mLGALS3, mLOXL3, mPLXND1, mOSR1, mRASSF2 originated from different sources: RASSF2 is a previously known marker, mOSR1 is from Epigenomics' discovery, the other five candidates were from in-silico discovery. All trained parameters were stored for use in the testing.

\section{Assay performance \\ HCCBloodTest: mSEPT9}

Valid HCCBloodTest results were reported for all patient samples in the test set (Table 2). Among the $60 \mathrm{HCC}$ cases, 46 tested positive, yielding a sensitivity of $76.7 \%$ (CI 95 64.6-85.6). Test results were also positive for 37 out of 103 cirrhotic patients without diagnosed HCC; observed specificity was $64.1 \%$ (CI 95 54.5-72.7).

A performance difference was observed among the various etiologies of chronic liver disease. For the 44 patients with chronic viral hepatitis-associated cirrhosis, the performance measures were $81.8 \%$ (18/22) sensitivity at $86.4 \%(19 / 22)$ specificity for HCC. For the 49 patients with NASH associated cirrhosis and $\mathrm{HCC}$, sensitivity was 
Table 1 Patient cohort characteristics of all enrolled patients including two that did not meet inclusion criteria for the final analysis

\begin{tabular}{|c|c|c|c|}
\hline Variable & Patient without HCC $(n=104)$ & Patients with HCC $(n=61)$ & \\
\hline Continuous & Median (min, max) & Median (min, max) & $p$ value (test method) \\
\hline Age (years) & $57(28-74)$ & $64(34-89)$ & $<0.001$ (Wilcox) \\
\hline Number of HCC nodules & - & $1(1-5)$ & - \\
\hline Size of largest HCC nodule (mm) & - & $27(7-118)$ & - \\
\hline Categorical & Count (\%) & Count (\%) & \\
\hline Male sex & $44(42.3)$ & $43(70.5)$ & $<0.001$ (Fisher) \\
\hline Child-class & & & 0.32 (Fisher) \\
\hline A & $60(57.7)$ & $41(67.2)$ & \\
\hline B & $43(41.3)$ & $20(32.8)$ & \\
\hline C (excluded) & $1(1.0)$ & & - \\
\hline Etiology of cirrhosis & & & 0.17 (Chi-square) \\
\hline ALD & $26(25.0)$ & $15(24.6)$ & \\
\hline HBV & $2(1.9)$ & $4(6.6)$ & \\
\hline $\mathrm{HCV}$ & $20(19.2)$ & $18(29.5)$ & \\
\hline NASH & $33(31.7)$ & $17(27.9)$ & \\
\hline Other & $23(22.1)$ & $7(11.5)$ & \\
\hline \multicolumn{4}{|l|}{ BCLC stage } \\
\hline Stage 0 & - & $10(16.4)$ & - \\
\hline Stage A & - & $39(63.9)$ & - \\
\hline Stage B & - & $11(18.0)$ & - \\
\hline Stage D (excluded) & & $1(1.6)$ & - \\
\hline
\end{tabular}

observed at $87.5 \%(14 / 16)$, but specificity was only $39.4 \%$ $(13 / 33)$.

\section{NGS panel}

For each patient sample a median of $204 \mathrm{k}$ NGS reads were obtained and assessed. The overall performance, as described by receiver operating characteristic curves found for testing (see Fig. 1), was similar to that observed from training. The AUC was 0.85 (95\% CI 0.78-0.91) and based on the trained algorithm we observed a sensitivity of $57 \%(95 \%$ CI $0.44-0.68)$ at $97 \%$ specificity (95\% CI 0.92-0.99), see Table 2. More granular data on performance by stage, etiology of cirrhosis and Child class status are provided in the Additional file 1: Table S1.

\section{Post hoc analyses}

As part of the cross-sectional study, patient data included AFP results. For comparative purposes AFP (at a cut off $\geq 20 \mathrm{ng}$ ) had a sensitivity of $36 \%$ at a specificity of $95 \%$. Further exploratory analyses were performed to assess the potential of multiomic panels including the methylation markers and AFP. Using the NGS marker panel in combination with AFP (logistic regression) lead to AUC of 0.9 (95\% CI $0.84-0.95)$ and sensitivity of $68 \%$ at $97 \%$ specificity.

\section{Discussion}

In the current cross-sectional study, we report on the performance of DNA methylation biomarkers isolated from cell free plasma DNA for the surveillance of patients with liver cirrhosis to detect HCC at an early stage. Cases comprised a cohort of patients with liver cirrhosis and early-stage HCC based on BCLC classification, representing the target surveillance population. Similarly, controls in the study comprised cirrhotic patients of the same Child classes, for whom no detectable HCC was present based on ultrasound imaging that was confirmed by MRI or CT.

In this Southern California population of patients, we observed an overall sensitivity of $76.7 \%$ at a specificity of $64.1 \%$ for mSEPT9 using the HCCBloodTest. These results are comparable to those reported by Kotoh et al. [18] for a mSEPT9 assay with a sensitivity of $62.5 \%$ for $\mathrm{HCC}$ at a specificity of $71.7 \%$ among cirrhotic patients. However, their study also included BCLC Stage C patients and enrolled exclusively in Japan, with a greater proportion of patients having chronic viral infection compared to our study. Similar to Kotoh et al., we observed improved HCC detection with more advanced stages of HCC. Our test sensitivity was also comparable to that reported by Oussalah et al. for BCLC Stage A patients (72.73\%), though the specificity 


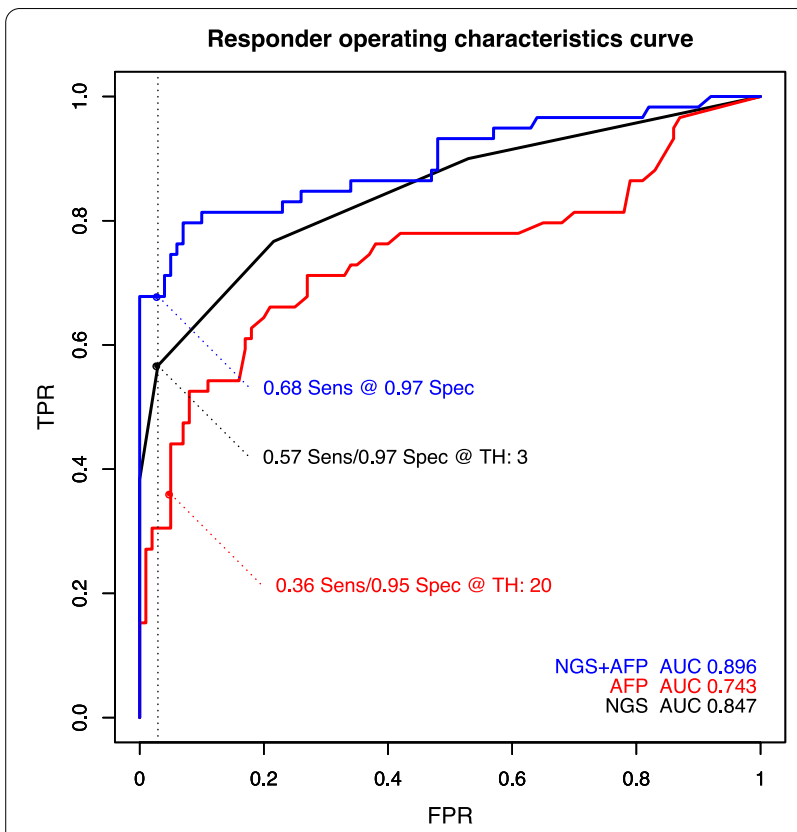

Fig. 1 Receiver operating characteristic (ROC) analysis of the NGS Panel, AFP and AFP + NGS Panel. ROC curves for the NGS panel (black), AFP (red) and combination of NGS panel with AFP (blue) using logistic regression for $60 \mathrm{HCC}$ versus 102 controls in the testing set. Areas under the curve (AUC) are written at the bottom right using the corresponding colors. Selected sensitivity/specificity pairs indicated on the curves are reported AFP $\geq 20 \mathrm{ng} / \mathrm{mL}$, the NGS panel using the trained algorithm, and the post hoc combination of the NGS panel and AFP

Table 2 Summary of sensitivity and specificity of surveillance methods for early stage HCC detection in patients with cirrhosis

\begin{tabular}{lll}
\hline Surveillance assay & Sensitivity & Specificity \\
\hline Test study & & \\
HCCBloodTest & $76.7 \%(\mathrm{Cl} 95$ 64.6-85.6) & $64.1 \%(\mathrm{Cl} 95: 54.5-72.7)$ \\
NGS panel & $57 \%(44-68)$ & $97 \%(92-99)$ \\
AFP (20 ng) & $36 \%(25-48)$ & $95 \%(89-98)$ \\
Post hoc analyses & & \\
AFP & $31 \%(20-43)$ & $97 \%(92-99)$ \\
mSEPT9+AFP & $42 \%(31-55)$ & $97 \%(92-99)$ \\
NGS panel+AFP & $68 \%(55-78)$ & $97 \%(92-99)$ \\
\hline
\end{tabular}

reported was higher in their study (86.4\%) [17]. While the underlying reasons for differences between the studies are not completely clear, and the sample size of the current study does not allow for detailed subgroup analysis, we did observe population differences. In the current study, there was a greater proportion of female subjects, fewer patients with BCLC stage B HCC and the spectrum of cirrhosis etiologies differed, with a greater proportion of NASH subjects in the non-HCC class. In this regard, we did observe substantial differences with respect to the specificity of the mSEPT9 assay, in particular lower performance in patients with NASH.

We also report on a panel of additional methylation biomarkers analyzed by bisulfite DNA NGS. These markers were initially selected and trained with an independent set of cases and controls, then tested using a fixed algorithm in a blinded fashion using the same patient collection as used for the HCCBloodTest. In this patient population the performance of the NGS panel showed improvement when compared with AFP. This outcome with epigenetic markers compares well with a recent report on hydroxymethylation where an AUC of 0.846 was observed for a 32-marker panel comparing patients with early stage $\mathrm{HCC}$ versus those with cirrhosis/chronic hepatitis [28]. Similarly, Hlady et al. [29] report good performance with a panel comprising hypo and hypermethylated $\mathrm{CpG}$ sites, though on a small sample size. Taken together, these reports suggest the potential for epigenetic markers measured in plasma cfDNA to aid in early detection of $\mathrm{HCC}$ in a surveillance program for patients with cirrhosis.

As part of a post-hoc analysis, we also combined the AFP outcomes with the methylated biomarker panel data to produce a multiomic panel which resulted in a sensitivity of $68 \%$ at a specificity of $97 \%$. These outcomes compare favorably with other reported methods discussed above. While clearly exploratory in nature, these data provide direction for future efforts to further improve the assay, and assess the marker combinations in a new independent test set. These data support the potential for this DNA methylation panel as a simple marker panel for early stage HCC detection. Performance compares well with other liquid biopsy assays. Exploratory inclusion of additional parameters such as age and sex, as outlined for GALAD, using logistic regression lead to increased AUCs but might be biased by over fitting within this limited data set.

The study had a few limitations. The sample size was limited, precluding a detailed sub-group analysis. However, the objective of this study was an assessment of methylation markers for early stage detection, and as such, the aggregate sample size was sufficient for this assessment. In addition, measurement of AFP-L3 and DCP were not available, limiting comparison to AFP but not the GALAD model. Finally, the cross-sectional design allows immediate assessment of the biomarkers for HCC detection. However, false positives in this design may resolve into true positives over time, if the markers are associated with the earliest stages of HCC development, that are not detectable by imaging. Nonetheless, 
the current design identified markers with surveillance potential for future analysis.

\section{Conclusions}

In this study, we demonstrated $57 \%$ detection of early stage HCC at an acceptable false positive rate of $3 \%$ for blood-based testing using methylation markers with a pretrained algorithm in an independent test set of well characterized cirrhotic patients with or without early stage HCC. This level of clinical performance can be achieved with a simple and affordable method and could be particularly applicable in settings where resources for surveillance by imaging may be limited. Furthermore, there is promise that the combination of the screening panel with other diagnostic parameters currently in use, such as AFP, may further enhance the performance. Though such findings require additional validation in an independent cohort, the results of this study support further development of cfDNA methylation markers for $\mathrm{HCC}$ surveillance in cirrhotic patients.

\begin{abstract}
Abbreviations
HCC: Hepatocellular carcinoma; AFP: Alpha fetoprotein; NGS: Next generation sequencing; BCLC: Barcelona clinic liver cancer staging system; AUC : Area under the curve; ctDNA: Circulating tumor DNA; cfDNA: Cell free DNA; bisDNA: Bisulfite treated DNA; mSEPT9: Methylated SEPTIN9; PCR: Polymerase chain reaction; $\mathrm{MRI}$ : Magnetic resonance imaging; CT: Computed tomography; NASH: Non-alcoholic steatohepatitis; ALD: Alcoholic liver disease; Cl: Confidence interval.
\end{abstract}

\section{Supplementary Information}

The online version contains supplementary material available at https://doi. org/10.1186/s12876-021-01714-8.

Additional file 1: The supplement includes study design details, further patient characteristics, and additional performance data.

\section{Acknowledgements}

The authors thank Dr. Fleischhacker for providing support to obtain samples for panel development and training and Innovis, LLC for study support for the sample collection in California.

\section{Authors' contributions}

J-experimental design, statistical analysis, manuscript preparation. DKexperimental design, study performance, data analysis, manuscript preparation. JA — study performance, sample management. TdV—experimental design, manuscript preparation. JG-experimental design, manuscript preparation. $\mathrm{OH}, \mathrm{SK}, \mathrm{FK}, \mathrm{PR}, \mathrm{SR}$ - study performance, data analysis. GW experimental design, statistical analysis, manuscript preparation. AZ-patient recruitment, sample collection and clinical data preparation, manuscript preparation. EM-patient recruitment, sample collection and clinical data preparation, manuscript preparation. TLF-experimental design, patient recruitment, sample collection and clinical data preparation, manuscript preparation. All authors have read and approved the manuscript.

\section{Funding}

The Study was sponsored by Epigenomics AG. The study was designed by the sponsor. The collection of samples and patient data was performed independently by the sites. The data was analyzed and interpreted by the sponsor in consultation with the site investigators. The manuscript was prepared by the sponsor in collaboration with the site investigators.

\section{Availability of data and materials}

The datasets used and/or analyzed during the current study are available from the corresponding author on reasonable request. Compiled data used for in silico discovery of the marker candidate panels assessed in this study comprised data sets that were obtained from the National Center for Biotechnology Information (NCBI) GEO database (https://www.ncbi.nlm.nih.gov/ geo/) (Edgar R, Domrachev M, Lash AE. "Gene Expression Omnibus: NCBI gene expression and hybridization array data repository" Nucleic Acids Res. 2002 Jan 1;30(1):207-10), thereof relevant for this study: Shimada et al., 2019, accession IDs GSE1 12791 and GSE1 13019; Horvath et al. 2014, accession ID GSE61258; accession ID GSE75041; Lehne et al. 2015 and Wahl et al. 2017 accession ID GSE55763; and Zaimi et al. 2018 accession ID GSE123914.

\section{Declarations}

\section{Ethics approval and consent to participate}

Samples from University Halle-Wittenberg, were collected with consent under ethical approval No. 2012-5. The study collection protocol was approved by the Institutional Review Boards at the University of Southern California (USC) Keck Medical Center and the California Liver Research Institute. At both sites and patients provided written informed consent prior to study participation.

\section{Consent for publication}

Not applicable.

\section{Competing interests}

Authors indicated by ${ }^{1,2}$ are full time employees of Epigenomics AG, the sponsor of the study.

\section{Author details}

${ }^{1}$ Epigenomics AG, Geneststr. 5, 10829 Berlin, Germany. ${ }^{2}$ Epigenomics Inc., 11055 Flintkote Ave, Suite A, San Diego, CA 92121, USA. ${ }^{3}$ Universitätsklinik und Poliklinik für Innere Medizin I, UKH Martin-Luther-Universität Halle-Wittenberg, Ernst-Grube-Str. 40, 06120 Halle (Saale), Germany. ${ }^{4}$ California Liver Research Institute, 301 S. Fair Oaks Avenue, Suite 409, Pasadena, CA 91105, USA. ${ }^{5}$ Keck School of Medicine, USC, 1510 San Pablo Street, 2/F, Los Angeles, CA 90033, USA.

Received: 24 September 2020 Accepted: 9 March 2021

Published online: 25 March 2021

\section{References}

1. Global Cancer Observatory. Cancer today. Estimated age-standardized incidence rates (World) in 2018, worldwide, both sexes, all ages. https:// gco.iarc.fr/today/online-analysis-multi-bars. Accessed 21 July 2020.

2. American Cancer Society. Key statistics about liver cancer. https:// www.cancer.org/cancer/liver-cancer/about/what-is-key-statistics.html. Accessed 21 July 2020

3. Centers for Disease Control and Prevention-Chronic Liver Disease and Cirrhosis. https://www.cdc.gov/nchs/fastats/liver-disease.htm. Accessed 21 July 2020.

4. Marrero JA, Kulik LM, Sirlin CB, Zhu AX, Finn RS, Abecassis MM, et al. Diagnosis, staging, and management of hepatocellular carcinoma: 2018 practice guidance by the american association for the study of liver diseases. Hepatology. 2018;68:723-50.

5. Tzartzeva K, Obi J, Rich NE, Parikh ND, Marrero JA, Yopp A, et al. Surveillance imaging and alpha fetoprotein for early detection of hepatocellular carcinoma in patients with cirrhosis: a meta-analysis. Gastroenterology. 2018;154(1706-1718):e1701.

6. Singal AG, Conjeevaram HS, Volk ML, Fu S, Fontana RJ, Askari F, et al. Effectiveness of hepatocellular carcinoma surveillance in patients with cirrhosis. Cancer Epidemiol Biomarkers Prev. 2012;21:793-9.

7. Parikh ND, Mehta AS, Singal AG, BlockT, Marrero JA, Lok AS. Biomarkers for the early detection of hepatocellular carcinoma. Cancer Epidemiol Biomark Prev. 2020;29(12):2495-503. 
8. Johnson PJ, Pirrie SJ, Cox TF, Berhane S, Teng M, Palmer D, et al. The detection of hepatocellular carcinoma using a prospectively developed and validated model based on serological biomarkers. Cancer Epidemiol Biomark Prev. 2014;23:144-53.

9. Yang JD, Addissie BD, Mara KC, Harmsen WS, Dai J, Zhang N, et al. GALAD score for hepatocellular carcinoma detection in comparison with liver ultrasound and proposal of GALADUS score. Cancer Epidemiol Biomark Prev. 2019;28:531-8.

10. Leygo C, Williams M, Jin HC, Chan MWY, Chu WK, Grusch M, et al. DNA methylation as a noninvasive epigenetic biomarker for the detection of cancer. Dis Markers. 2017;2017:3726595.

11. Sun K, Jiang P, Chan KC, Wong J, Cheng YK, Liang RH, et al. Plasma DNA tissue mapping by genome-wide methylation sequencing for noninvasive prenatal, cancer, and transplantation assessments. Proc Natl Acad Sci U S A. 2015;112:E5503-5512.

12. Moss J, Magenheim J, Neiman D, Zemmour H, Loyfer N, Korach A, et al. Comprehensive human cell-type methylation atlas reveals origins of circulating cell-free DNA in health and disease. Nat Commun. 2018;9:5068.

13. Mandel P, Metais P. Les acides nucleiques du plasma sanguin chez I'homme. C R Acad Sci III 1947;112.

14. Thierry AR, El Messaoudi S, Gahan PB, Anker P, Stroun M. Origins, structures, and functions of circulating DNA in oncology. Cancer Metastasis Rev. 2016;35:347-76.

15. Akuta N, Kawamura Y, Kobayashi M, Arase Y, Saitoh S, Fujiyama S, et al. TERT promoter mutation in serum cell-free DNA is a diagnostic marker of primary hepatocellular carcinoma in patients with nonalcoholic fatty liver disease. Oncology. 2020;99:1-10.

16. Grutzmann R, Molnar B, Pilarsky C, Habermann JK, Schlag PM, Saeger $H D$, et al. Sensitive detection of colorectal cancer in peripheral blood by septin 9 DNA methylation assay. PLoS ONE. 2008;3:e3759.

17. Oussalah A, Rischer S, Bensenane M, Conroy G, Filhine-Tresarrieu P, Debard R, et al. Plasma mSEPT9: a novel circulating cell-free DNA-based epigenetic biomarker to diagnose hepatocellular carcinoma. EBioMedicine. 2018;30:138-47.

18. Kotoh Y, Suehiro Y, Saeki I, Hoshida T, Maeda M, Iwamoto T, et al. Novel liquid biopsy test based on a sensitive methylated SEPT9 assay for diagnosing hepatocellular carcinoma. Hepatol Commun. 2020;4:461-70.
19. Hall PA, Russell SE. The pathobiology of the septin gene family. J Pathol. 2004;204:489-505.

20. Epi BiSKit Instructions for Use, IFU 0019GB, rev1. 05 February2018, Epigenomics AG.

21. Rausch S, Hasinger O, Konig T, Schlegel A, Weiss G. An automated high throughput solution for DNA extraction and bisulfite-conversion from high volume liquid biopsy specimens: sample preparation for epigenetic analysis. BMC Res Notes. 2019;12:551.

22. Lewin J, Plum A, Hildmann T, Rujan T, Eckhardt F, Liebenberg V, et al. Comparative DNA methylation analysis in normal and tumour tissues and in cancer cell lines using differential methylation hybridisation. Int J Biochem Cell Biol. 2007;39:1539-50.

23. Magoc T, Salzberg SL. FLASH: fast length adjustment of short reads to improve genome assemblies. Bioinformatics. 2011;27:2957-63.

24. R Core Team. $R$ : a language and environment for statistical computing. $R$ Foundation for Statistical Computing. Vienna (2018). https://www.R-proje ct.org/

25. Eddelbuettel D, Francois R. Rcpp: seamless R and C++ integration. J Stat Softw. 2011;40:1-18.

26. Eckhardt F, Lewin J, Cortese R, Rakyan VK, Attwood J, Burger M, et al. DNA methylation profiling of human chromosomes 6, 20 and 22. Nat Genet. 2006;38:1378-85.

27. HCC BloodTest Instructions for Use, IFU 0021, rev 2. 15October 2018, Epigenomics AG

28. Cai J, Chen L, Zhang Z, Zhang X, Lu X, Liu W, et al. Genome-wide mapping of 5-hydroxymethylcytosines in circulating cell-free DNA as a noninvasive approach for early detection of hepatocellular carcinoma. Gut. 2019;68:2195-205.

29. Hlady RA, Zhao X, Pan X, Yang JD, Ahmed F, Antwi SO, et al. Genomewide discovery and validation of diagnostic DNA methylation-based biomarkers for hepatocellular cancer detection in circulating cell free DNA. Theranostics. 2019;9:7239-50.

\section{Publisher's Note}

Springer Nature remains neutral with regard to jurisdictional claims in published maps and institutional affiliations.
Ready to submit your research? Choose BMC and benefit from:

- fast, convenient online submission

- thorough peer review by experienced researchers in your field

- rapid publication on acceptance

- support for research data, including large and complex data types

- gold Open Access which fosters wider collaboration and increased citations

- maximum visibility for your research: over $100 \mathrm{M}$ website views per year

At BMC, research is always in progress.

Learn more biomedcentral.com/submissions 